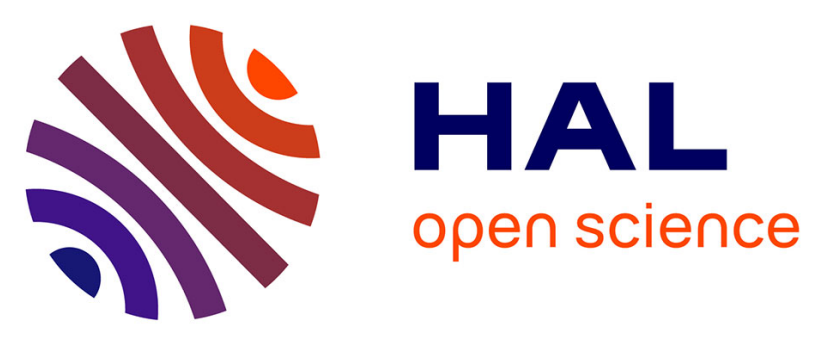

\title{
Enhanced Chemical Synthesis at Soft Interfaces: A Universal Reaction-Adsorption Mechanism in Microcompartments
}

Ali Fallah-Araghi, Kamel Meguellati, Jean-Christophe Baret, Abdeslam El Harrak, Thomas Mangeat, Martin Karplus, Sylvain Ladame, Carlos M. Marques, Andrew D. Griffiths

\section{To cite this version:}

Ali Fallah-Araghi, Kamel Meguellati, Jean-Christophe Baret, Abdeslam El Harrak, Thomas Mangeat, et al.. Enhanced Chemical Synthesis at Soft Interfaces: A Universal Reaction-Adsorption Mechanism in Microcompartments. Physical Review Letters, 2014, 112 (028301), pp.0283301(1-5). 10.1103/PhysRevLett.112.028301 . hal-01122849

\section{HAL Id: hal-01122849 https://hal.science/hal-01122849}

Submitted on 3 May 2019

HAL is a multi-disciplinary open access archive for the deposit and dissemination of scientific research documents, whether they are published or not. The documents may come from teaching and research institutions in France or abroad, or from public or private research centers.
L'archive ouverte pluridisciplinaire HAL, est destinée au dépôt et à la diffusion de documents scientifiques de niveau recherche, publiés ou non, émanant des établissements d'enseignement et de recherche français ou étrangers, des laboratoires publics ou privés. 


\title{
Enhanced Chemical Synthesis at Soft Interfaces: A Universal Reaction-Adsorption Mechanism in Microcompartments
}

\author{
Ali Fallah-Araghi, ${ }^{1}$ Kamel Meguellati, ${ }^{1}$ Jean-Christophe Baret, ${ }^{2, *}$ Abdeslam El Harrak, ${ }^{3}$ Thomas Mangeat, ${ }^{3}$ \\ Martin Karplus, ${ }^{1,4}$ Sylvain Ladame, ${ }^{1,5,}$ Carlos M. Marques, ${ }^{6, \$}$ and Andrew D. Griffiths ${ }^{1,7, \S}$ \\ ${ }^{1}$ Institut de Sciences et d'Ingénierie Supramoléculaires (ISIS), Université de Strasbourg, \\ CNRS UMR 7006, 8 allée Gaspard Monge, F-67083 Strasbourg Cedex, France \\ ${ }^{2}$ Max Planck Institute for Dynamics and Self-organization, Am Fassberg 17, D-37077 Goettingen, Germany \\ ${ }^{3}$ Raindance Technologies France, 8 allée Gaspard Monge, F-67083 Strasbourg Cedex, France \\ ${ }^{4}$ Department of Chemistry and Chemical Biology, 12 Oxford Street, Harvard University, \\ Cambridge, Massachussets, Massachusetts 02138, USA \\ ${ }^{5}$ Department of Bioengineering, Imperial College London, London SW72AZ, United Kingdom \\ ${ }^{6}$ Institut Charles Sadron, Université de Strasbourg, CNRS UPR 22, 23 rue du Loess, F-67034 Strasbourg Cedex, France \\ ${ }^{7}$ École Supérieure de Physique et de Chimie Industrielles de la Ville de Paris (ESPCI ParisTech), \\ CNRS UMR 7084, 10 rue Vauquelin, F-75231 Paris Cedex 05, France
}

(Received 29 July 2013; revised manuscript received 10 October 2013; published 13 January 2014)

A bimolecular synthetic reaction (imine synthesis) was performed compartmentalized in micrometerdiameter emulsion droplets. The apparent equilibrium constant $\left(K_{\text {eq }}\right)$ and apparent forward rate constant $\left(k_{1}\right)$ were both inversely proportional to the droplet radius. The results are explained by a noncatalytic reaction-adsorption model in which reactants adsorb to the droplet interface with relatively low binding energies of a few $k_{B} T$, react and diffuse back to the bulk. Reaction thermodynamics is therefore modified by compartmentalization at the mesoscale — without confinement on the molecular scale-leading to a universal mechanism for improving unfavorable reactions.

DOI: 10.1103/PhysRevLett.112.028301

PACS numbers: $82.70 . \mathrm{Kj}, 68.05 .-\mathrm{n}, 82.60 .-\mathrm{s}$

Entropic factors tend to disfavor synthetic chemical reactions: the formation of one product molecule from two reactant molecules results in a high loss of mobility (and hence a loss of entropy) [1]. This thermodynamic unfavorability has, for example, led to strong criticism [2-4] of the prebiotic broth theory for the origin of life of Darwin, Oparin, and Haldane [5-7] as prebiotic concentrations of organic building blocks seem too low for a self-replicating, evolvable system to arise [8]. Mesoscale compartmentalization may have played an important role in overcoming this thermodynamic problem. Microcompartments of $\sim 1-100 \mu \mathrm{m}$ diameter in hydrothermal vents, while not altering the thermodynamics of individual reactions, are proposed to have limited diffusion of reaction products (which then participate in further reactions) [9], while evaporation of $\sim 1 \mu \mathrm{m}$ diameter atmospheric aerosol droplets is proposed to have actively concentrated reactants [10]. Indeed, a wide range of organic compounds has been identified in present-day secondary aerosol particles, including higher molecular weight products thought to be synthesized in the condensed phase; however, the synthetic mechanism is still poorly understood [11]. The kinetics of many chemical reactions can also be improved by employing catalysts that function by confinement in nanosized environments, such as nanopores in zeolites. However, entropic confinement effects are observed only when the system is confined at the molecular scale and the pores are of similar size $(\lesssim 2 \mathrm{~nm})$ and shape to the reactant(s), transition state(s), and product(s) [12-14].

We demonstrate here that both the kinetics and thermodynamics of synthetic reactions can be enhanced by compartmentalization at the mesoscale, in micrometer-diameter droplets, a feature considered as a sharp advantage in "prevolutionary dynamics" [15]. The process is based on an interfacial reaction-diffusion mechanism that is expected to be quite general, as it requires no size or shape complementarity and only relatively low energy binding of the reactants to the droplet interface.

As a model system, we used the reversible reaction of a nonfluorescent amine 1 with a very weakly fluorescent aldehyde 2 to form a fluorescent imine 3 in water (Fig. 1, Supplemental Material Fig. 1) [16,17]. For simplicity in the following, the reactants are considered the same $(A)$ and the product $(A A)$. We study the role of compartmentalization mediated by soft liquid-liquid interfaces on the efficiency of thermodynamically controlled reactions.

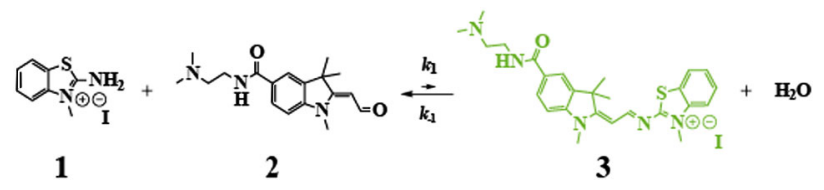

FIG. 1 (color online). Model chemical reaction: a fluorescent imine $(A A)$ is synthesized from two nonfluorescent reagents (A) (Supplemental Material). 
We used microfluidic emulsification to produce monodisperse compartments made of aqueous droplets of 2.5 to $160 \mathrm{pL}$ volume (radius $R$ from 8 to $34 \mu \mathrm{m}$ ) [18] in a fluorinated oil and stabilized by a nonionic surfactant. The surfactant was a triblock copolymer of two perfluoropolyether (PFPE) chains linked by one polyethyleneglycol (PEG) chain [19] and used at a concentration of $2.37 \mathrm{mM}$ $(2 \% \mathrm{w} / \mathrm{w})$ in the oil (Supplemental Material, Figs. 2-3 and Supplemental Table 1).

For droplets containing $15 \mathrm{mM}$ of the reactants, the concentration of the product was increasing when the size of the droplet decreased (Fig. 2). Although the reaction kinetics in bulk were exponential, in droplets the reaction kinetics were slightly sigmoidal, indicating a mechanism with at least two steps $[20,21]$. Both the apparent $k_{1}$ and the apparent $K_{\text {eq }}$ were inversely related to the droplet radius, $R$ (Fig. 3 and Supplemental Material Fig. 4). In $2.5 \mathrm{pL}$ droplets the apparent $k_{1}=8.2 \times 10^{-4} \mathrm{M}^{-1} \mathrm{~s}^{-1}$ (calculated using the maximum rate) and the apparent $K_{\text {eq }}=0.78 \mathrm{M}^{-1}$ were 45 -fold and 29 -fold higher than $k_{1}$ and $K_{\text {eq }}$ in bulk. The apparent $k_{-1}$ was similar in bulk solvent and in droplets of all sizes [Fig. 3(c)]. Finally, the fluorescent product was located inside the droplet, with no detectable accumulation at the water-oil interface (Fig. 3(d), Supplemental Fig. 4). The standard Gibbs free energies of the reactants $R c$, transition state $T S$, and product $P r$ were calculated from the apparent $K_{\text {eq }}, k_{1}$, and $k_{-1}$ [Fig. 3(e)] [22-24]. Imine 3 synthesis becomes more favorable in droplets than in bulk largely due to the decrease in $G_{P r}^{0}-G_{R c}^{0}$, whereas there is almost no change in $G_{P r}^{0}-G_{T S}^{0}$, indicating that stabilization of the transition state plays, at most, a minor role.

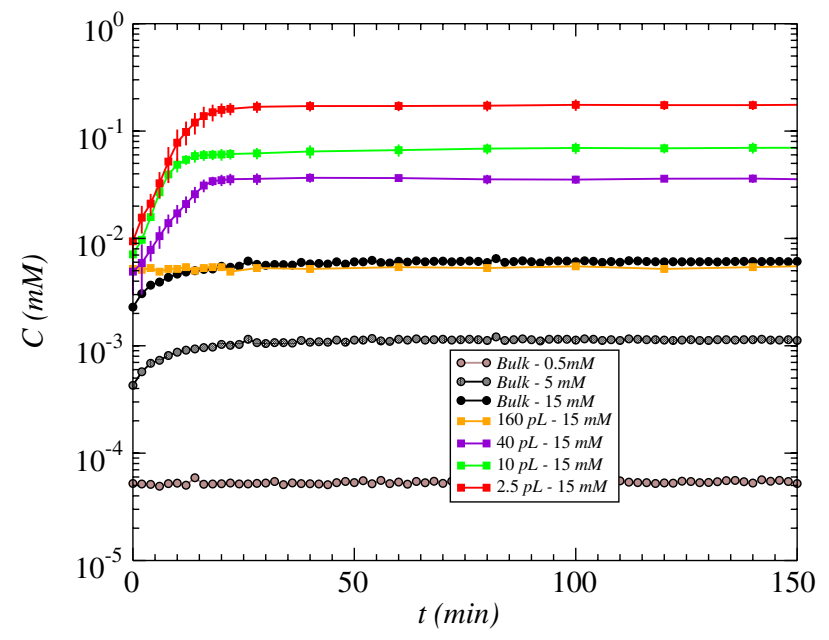

FIG. 2 (color online). Concentration $C$ of imine 3 product as a function of time in bulk and in droplets. Stoichiometric mixtures of amine 1 and aldehyde 2 were used at the concentrations indicated. The initial rate and equilibrium constant of the reaction are significantly increasing when the reaction occurs in droplets of decreasing sizes (from 160 to $2.5 \mathrm{pL}$; Supplemental Tables 1-3). Error bars correspond to \pm 1 standard deviation.
We excluded a series of phenonema potentially involved in the mechanism of the reaction enhancement. First, our observations cannot be explained by the droplets functioning as optical resonators since a stable trimethine cyanine dye Cy3 was no more fluorescent when emulsified than in bulk [Supplemental Fig. 5(a)]. Second, the thermodynamics of the reaction is not affected by the Laplace pressure: in $2.5 \mathrm{pL}$ droplets the Laplace pressure $\gamma / R \approx$ $500 \mathrm{~Pa} \ll 1$ bar ( $\gamma$ being the interfacial tension). Third, partitioning in the oil is not relevant, as when the reactants were encapsulated separately in a mixed emulsion, the fluorescence was not increasing over ten hours, indicating no exchange of the reactants through the oil [25] [Supplemental Fig. 5(b)]. Finally, we observed differences
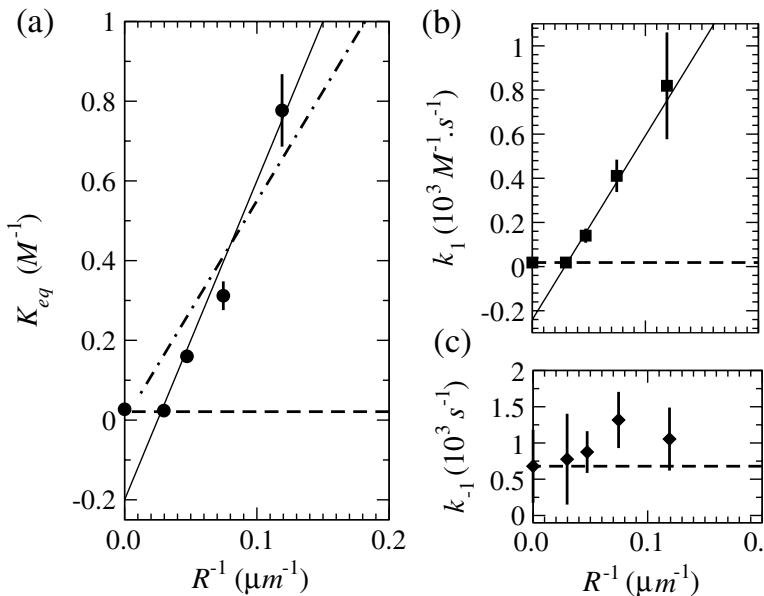

(c)

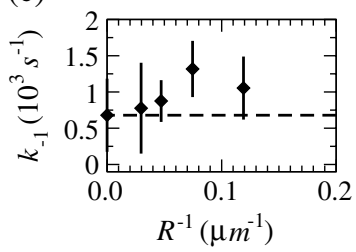

(d)
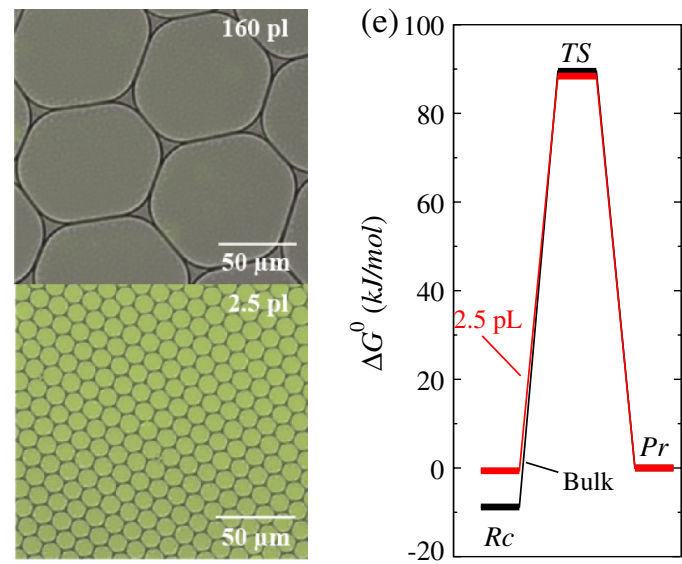

FIG. 3 (color online). Equilibrium constant for the reaction in droplets (a), forward (b), and backward (c) rate constants of the reaction as a function of droplet size. The dashed lines correspond to bulk values. The dotted and dashed line correspond to a $1 / R$ relationship recovered in the model [Eq. (8)]. The direct fit by linear regression of the droplet data (solid line) shows a negative intercept with the $y$ axis, which is explained by the solubility of the product in the oil phase. Error bars correspond to \pm 1 standard deviation. (d) Fluorescence imaging of 2.5 and $160 \mathrm{pL}$ droplets, showing the increase of product in the small droplets. (e) Thermodynamics of imine formation in $2.5 \mathrm{pL}$ droplets compared to bulk (Supplemental Table 2). 
in reaction efficiency between batches of surfactant in droplets. We speculated that residual unreacted carboxylate head groups of the PFPE Krytox FSH (DuPont) used in surfactant synthesis [19] may lead to the interaction with both the cationic reactants and product at the interface [17]. However, using a mixture of $0.2 \% \mathrm{w} / \mathrm{w}$ of the triethylamine salt of Krytox FSH (with free carboxylate) and $1.8 \% \mathrm{w} / \mathrm{w}$ surfactant did not result in further enhancement of the reaction (compared to $2 \% \mathrm{w} / \mathrm{w}$ surfactant alone), setting up an upper bound for the potential contamination with residual Krytox.

To explain our experimental results, we developed a minimal model combining the well-known thermodynamic concepts for bulk and surface bimolecular reactions with those for surface adsorption [26]. We show how bulk reactions in small droplets with finite radius $R$ can be strongly influenced by reaction and adsorption phenomena at the droplet surface.

The thermodynamic cycle in droplets is governed by four reversible processes: (1) Reaction $A+A \rightleftarrows A A$ in bulk, (2) binding of reactants to the droplet interface, (3) reaction $A+A \rightleftarrows A A$ at interface, and (4) dissociation of product $A A$ from the interface (Fig. 4(a),(b), Supplemental Material [27]). Using $\sigma_{A}, \sigma_{A A}$ as the surface concentrations of $A$ and $A A$ and $c_{A}$ and $c_{A A}$ as their bulk concentrations, mass conservation for a total number $N_{A}$ of molecules $A$ reads

$$
4 \pi R^{2}\left(\sigma_{A}+2 \sigma_{A A}\right)+\int_{0}^{R} 4 \pi r^{2} d r\left[c_{A}(r)+2 c_{A A}(r)\right]=N_{A} .
$$

By assuming that species $A$ and $A A$ have similar diffusion coefficients, $D=D_{A}=D_{A A}$, the bimolecular reactions inside the droplet and at interface are expressed as

$$
\begin{gathered}
\partial_{t} c_{A}=2 k_{-1}^{b} c_{A A}-2 k_{1}^{b} c_{A}^{2}+D \Delta c_{A} \\
\partial_{t} c_{A A}=-k_{-1}^{b} c_{A A}+k_{1}^{b} c_{A}^{2}+D \Delta c_{A A} \\
\partial_{t} \sigma_{A}=2 k_{-1}^{s} \sigma_{A A}-2 k_{1}^{s} \sigma_{A}^{2}-\left.D \nabla c_{A}\right|_{r=R} \\
\partial_{t} \sigma_{A A}=-k_{-1}^{s} \sigma_{A A}+k_{1}^{s} \sigma_{A}^{2}-\left.D \nabla c_{A A}\right|_{r=R},
\end{gathered}
$$

where $\partial_{t}$ is the time derivative and $\Delta$ is the radial component of the diffusion operator in spherical coordinates and $k_{-1}^{b, s}$ and $k_{1}^{b, s}$ the two kinetic coefficients associated with the reaction in bulk $\left({ }^{b}\right)$ or at the interface $\left({ }^{s}\right)$. In an infinite system, the reaction equilibrium constant is $K_{\mathrm{eq}}^{b}=c_{A A}^{\mathrm{eq}} /\left(c_{A}^{\mathrm{eq}}\right)^{2}=$ $k_{1}^{b} / k_{-1}^{b}$. At an isolated interface, $K_{\mathrm{eq}}^{s}=\sigma_{A A}^{\mathrm{eq}} /\left(\sigma_{A}^{\mathrm{eq}}\right)^{2}=$ $k_{1}^{s} / k_{-1}^{s}$. The last terms on the rhs of Eqs. (4) and (5) account for the net exchange fluxes between the surface and the bulk. For surface densities far from saturation, these fluxes can be written as

$$
\begin{aligned}
-\left.D \nabla c_{A}\right|_{r=R} & =k_{A}^{b s} c_{A}(R)-k_{A}^{s b} \sigma_{A} \\
-\left.D \nabla c_{A A}\right|_{r=R} & =k_{A A}^{b s} c_{A A}(R)-k_{A A}^{s b} \sigma_{A A}
\end{aligned}
$$

so that in the absence of any surface and bulk reactions, $\left.\nabla c_{A}\right|_{r=R}=0,\left.\nabla c_{A A}\right|_{r=R}=0$, the bulk and surface densities assume the usual nonsaturated values of the Langmuir isotherm [26],

$$
\begin{aligned}
\sigma_{A} & =k_{A}^{b s} / k_{A}^{s b} c_{A}=\delta_{A} c_{A} \\
\sigma_{A A} & =k_{A A}^{b s} / k_{A A}^{s b} c_{A A}=\delta_{A A} c_{A A},
\end{aligned}
$$

with the conventional surface to bulk and bulk to surface rate constants $k_{A}^{b s}, k_{A}^{s b}, k_{A A}^{b s}, k_{A A}^{s b}$. The lengths $\delta_{A}=k_{A}^{b s} / k_{A}^{s b}$ and $\delta_{A A}=k_{A A}^{b s} / k_{A A}^{s b}$, known as the adsorption lengths [28], can span a wide range of values from a few nanometers for weakly adsorbing species to macroscopic values for strong surface affinities. Contrary to adsorption phenomena of nonreactive species, adsorption and desorption induces in this case gradients of substrate $A$ and product $A A$ molecules close to the interface. More specifically, product molecules $A A$ generated at the surface can desorb and enrich, before decaying, the immediate vicinity of the interface, over a characteristic length $\xi$ determined by the diffusion coefficient $D$ and the decay time $k_{-1}^{b}$ of the molecules, $\xi^{2}=D / k_{-1}^{b}$ [Fig. 4(b),(c)]. In the experiments, $k_{-1}^{b}=1.06 \times 10^{-3} \mathrm{~s}^{-1}$, $D=2.39 \times 10^{-10} \mathrm{~m}^{2} \mathrm{~s}^{-1}, \xi=476 \mu \mathrm{m}>R$. In the limit where $R \ll \xi$, the desorbed product molecules quickly redistribute in the droplet resulting in a homogeneous fluo- (a)

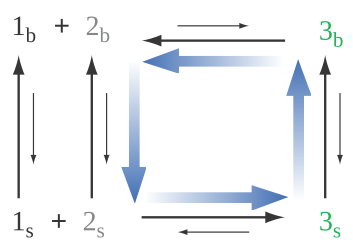

(c)

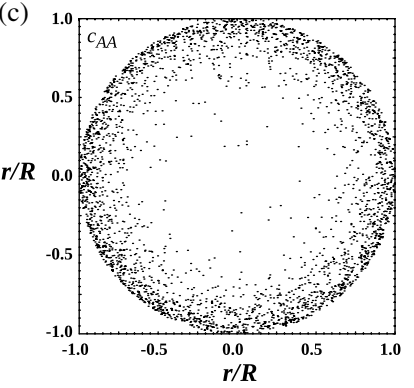

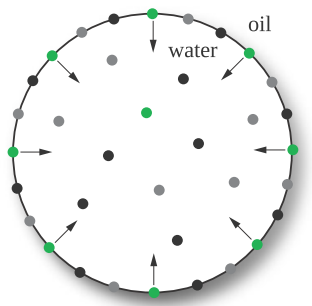

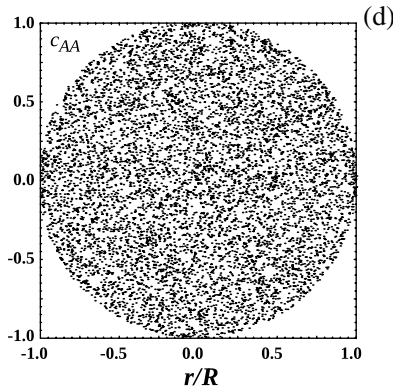

(b)

FIG. 4 (color online). (a) Model scheme for the reaction including surface interactions. Reactants and products in bulk and at the surface are subscripted $\mathrm{b}$ and $\mathrm{s}$, respectively. The net flux is shown by blue arrows. (b) Scheme of the diffusion process for the products towards the bulk of a droplet of radius $R$. (c,d) Concentration profiles were obtained by solving the adsorptionreaction-diffusion equation for two limiting cases $R / \xi=0.1$ (c) and $R / \xi=10$ (d) $\left(\xi=\sqrt{D / k_{-1}}\right)$. 
rescence in the droplet, as seen in the experiments (Fig. 3). In this limit, the reaction-adsorption model can be solved analytically (see Supplemental Material [27] for the derivation): for small droplets $R \ll \xi$ and relatively low surface affinities $\left(\delta_{A, A A} \ll R\right)$ of the substrate and product, the surface mediated reaction is of second order and with an effective reaction constant,

$$
K_{\mathrm{eq}}^{\mathrm{eff}}=\frac{\left\langle c_{A A}\right\rangle}{c^{2}}=K_{\mathrm{eq}}^{s} \frac{3 \delta_{A}^{2}}{R}\left(\frac{k_{-1}^{b}}{k_{-1}^{s}}+\frac{k_{-1}^{b}}{k_{A A}^{s b}}\right)^{-1},
$$

where $\left\langle c_{A A}\right\rangle$ and $c$ are the average concentrations of products and reactants, respectively. Equation (8) first shows that the reaction in droplets is-as in bulk-second order (an order of 1.88 was measured experimentallySupplemental Table 3). This also implies that the system is in the linear phase of the Langmuir isotherm, where the surface is unsaturated [26], consistent with (i) the relatively low affinity interaction of the reactants with the interface and (ii) the fact that the rate limiting step of the reaction is chemical [29]. Second, Eq. (8) displays a $1 / R$ dependence, qualitatively recovered in the experiments $R K_{\text {eq }}=5.5 \pm 2.5 \times 10^{-6} \mathrm{~m} \cdot \mathrm{M}^{-1} \quad$ [Fig. 3(a)]. Quantitatively, the experiments show a slope $\partial K_{\mathrm{eq}} / \partial(1 / R) \approx$ $8.3 \times 10^{-6} \mathrm{M}^{-1} \mathrm{~m}$ that can be used to estimate the binding energy $\varepsilon_{A}$ of the reactants to the interface in the limit of fast product desorption $\left(k_{A A}^{s b} \gg k_{-1}^{s}\right)$ [30],

$$
\epsilon_{A}=k_{B} T \ln \left(\delta_{A} / a\right)=\frac{k_{B} T}{2} \ln \left(\frac{1}{3 a K_{\mathrm{eq}}^{b}} \frac{d K_{\mathrm{eq}}^{\mathrm{eff}}}{d 1 / R}\right),
$$

with a molecular length $a=1 \mathrm{~nm}, \quad \varepsilon_{A} \sim 5.7 k_{B} T$ $\left(14 \mathrm{~kJ} \cdot \mathrm{mol}^{-1}\right)$. This relatively low binding energy is equivalent to about five van der Waals interactions. It is the (negative) free energy of binding of the reactants to the interface that compensates the increase in $G_{R c}^{0}$ observed in droplets compared to in bulk [Fig. 3(e)]. The model is also self-consistent as the affinity length $\delta_{A}=0.1 \mu \mathrm{m} \ll$ $R$ as initially hypothesized. Finally, the negative intercept of $K_{\text {eq }}$ as a function of $1 / R$ with the $y$ axis $\left(-0.25 \mathrm{M}^{-1}\right)$ is attributed to product sequestration in the micellar fraction. Assuming that any imine 3 in the micellar fraction is nonfluorescent, we estimate the amount of product in the oil $c_{A A}^{\mathrm{mic}}$ using the fact that, at equilibrium, only a small fraction of the reactants is transformed to product, $c_{A A}^{\mathrm{mic}} \simeq-K_{\mathrm{eq}}^{\mathrm{eff}} c_{A}^{2} \sim 56 \mu \mathrm{M}$.

Interestingly, it has previously been shown that several reactions are greatly accelerated when carried out in vigorously stirred aqueous suspensions with high interfacial area [31]. Here, comparing the energies involved provides interesting insights: in $2.5 \mathrm{pL}$ droplets the chemical equilibrium is shifted by $\sim 10 \mathrm{~kJ} \cdot \mathrm{mol}^{-1}$ which corresponds to a total chemical energy of $10^{-12} \mathrm{~J}$ with a concentration of $\sim 0.17 \mathrm{mM}$ of product. The mechanical work done to create the interface of the $2.5 \mathrm{pL}$ droplet is $W=4 \pi \gamma R^{2} \sim 10^{-12} \mathrm{~J}$, the same order of magnitude as the chemical energy. The improvement in reaction efficiency in droplets apparently comes from the transformation of the mechanical energy used to form droplets into chemical energy which might also provide a mechanistic basis for the acceleration seen with the "on water" reactions that has so far not been elucidated.

The reaction-diffusion-adsorption mechanism described here has a number of interesting features. The mechanism is noncatalytic, reaction thermodynamics being shifted in favor of product formation in droplets compared to in bulk solvent (water). It also relies on compartmentalizations at the mesoscale in micrometer-diameter droplets rather than in nanosized environments, such as nanopores in zeolites [12-14] or micelles [32,33]. However, compared to catalysis by confinement at the molecular scale-as is the case with zeolite catalysts-we expect the mechanism we describe to be quite general, requiring no size or shape complementarity and only relatively low energy binding of the reactants to the droplet interface. There are, nevertheless, some similarities with the enhancement of certain reactions in micellar systems. In some cases, micelles function as catalysts (reduction of the activation energy) while in other cases the enhancement is thought to be due to the micellar microenvironment (activity coefficient effects). However, for bimolecular reactions, the dominant effect in many cases is thought to be due to reactants being concentrated relative to the surrounding water phase through interaction with the micelle surface or insertion into the micelle itself (entropy effects) [32,33] as we observed for the droplet system.

Micrometer-size compartments are ubiquitous in the environment. In particular, aerosol droplets are present in massive quantities in the atmosphere and have a very important impact on atmospheric processes, climate, and health [34]. The mechanism we describe could help to explain the organic mass in present-day atmospheric secondary aerosols (formed in the atmosphere by condensation of atmospheric gases) [34,11]. The operation of this mechanism in ancient aerosol droplets [10], or other micron-scale spaces, such as those found in hydrothermal vents [9], may also help to explain how, despite the inherent thermodynamic unfavorability of synthetic reactions, a sufficient level of prebiotic synthetic chemistry was achieved for autocatalytic systems capable of self-replication and evolution to appear and life to begin [15]. Finally, our results could also have implications in synthetic biology approaches for the design, creation, and control of minimal biomimetic systems having specific synthetic functions [35,36].

In summary, we have shown that the efficiency of a synthetic reaction can be enhanced in picolitre volume droplets. The results can be explained by a simple reaction-adsorption mechanism coupling bulk and surface reactions that requires only relatively low binding energies of a few $k_{B} T$ (equivalent to just a few van der Waals 
interactions) of reactants to the droplet interface. The reaction thermodynamics are shifted in favor of product formation in droplets compared to in bulk solvent controlled by the dimensionless number $\sqrt{D /\left(k_{-1} R^{2}\right)}$, comparing the typical distance over which a product molecule diffuses in the time given by the reverse rate constant in bulk solvent to the compartment size. For large values of this dimensionless number ("small" droplet sizes), the equilibrium constant and the forward reaction rate both scale as $1 / R$, leading to a strong increase in synthetic efficiency at small dimension, a phenomenon of particular relevance for prebiotic chemistry, atmospheric aerosol chemistry, and synthetic biology.

This work was supported by the Ministère de l'Enseignement Supérieur et de la Recherche and the Centre National de la Recherche Scientifique (CNRS). J.-C. B. acknowledges support by funding from the European Research Council (ERC) under the European Union's Seventh Framework Program (FP7/2007-2013)/ ERC Grant No. 306385-SofI. We thank J. Baudry and J. Bibette for helpful discussions, Y. Beck for preparation of streptavidin coated beads, G. Koripelly for the synthesis of $\mathrm{Cy} 3$, J. Mutterer for help with microscopy, and Q. Brosseau for surface tension and DLS measurements. A. F.-A. and K. M. contributed equally to this work.

*jean-christophe.baret@ds.mpg.de

†s.ladame@imperial.ac.uk

*marques@unistra.fr

§andrew.griffiths@espci.fr

[1] M. I. Page and W. P. Jencks, Proc. Natl. Acad. Sci. U.S.A. 68, 1678 (1971).

[2] A. Cairns-Smith, Genetic Takeover and the Mineral Origin of Life (Cambridge University Press, New York, 1982).

[3] C. Thaxton, W. Bradley, and R. Olsen, The Mystery of Life's Origin (Philosophical Library Inc., New York, 1984).

[4] C. Woese, J. Mol. Evol. 13, 95 (1979).

[5] F. Darwin, The Life and Letters of Charles Darwin (John Murray, London, 1887).

[6] J. B. S. Haldane, Rationalist Annu. 148, 3 (1929).

[7] A. Oparin, Proiskhozhdenie zhizny (Izd. Mosk. Rabochii, Moscow, 1924).

[8] C. de Duve, Blueprint for a Cell: The Nature and Origin of Life (Neil Paterson Publisher, Burlington, North Carolina, 2003).

[9] E. V. Koonin and W. Martin, Trends Genet. 21, 647 (2005).

[10] C. M. Dobson, G. B. Ellison, A. F. Tuck, and V. Vaida, Proc. Natl. Acad. Sci. U.S.A. 97, 11864 (2000).
[11] M. Hallquist, J. Wenger et al., Atmos. Chem. Phys. 9, 5155 (2009).

[12] B. Smit and T. L. M. Maesen, Nature (London) 451, 671 (2008).

[13] H. J. G. E. Gardeniers, Anal. Bioanal. Chem. 394, 385 (2009).

[14] R. Gounder and E. Iglesia, Chem. Commun. (Cambridge) 49, 3491 (2013).

[15] I. A. Chen and M. A. Nowak, Acc. Chem. Res. 45, 2088 (2012).

[16] K. Meguellati, M. Spichty, and S. Ladame, Org. Lett. 11, 1123 (2009).

[17] K. Meguellati, A. Fallah-Araghi, J.-C. Baret, A. E. Harrak, T. Mangeat, M. Karplus, S. Ladame, C. Marques, and A. D. Griffiths; Chem. Commun. (Cambridge) 49, 11332 (2013).

[18] S. L. Anna, N. Bontoux, and H. A. Stone, Appl. Phys. Lett. 82, 364 (2003).

[19] C. Holtze, A. C. Rowat, J. J. Agresti, J. B. Hutchison, F. E. Angile, C. H. J. Schmitz, S. Koester, H. Duan, K. J. Humphry, R. A. Scanga et al., Lab Chip 8, 1632 (2008).

[20] E. Boecker, Experientia 40, 453 (1984).

[21] K. Connors, Chemical Kinetics: The Study of Reaction Rates in Solution (Wiley, New York, 1990).

[22] M. Evans and M. Polanyi, Trans. Faraday Soc. 31, 875 (1935).

[23] H. Eyring, J. Chem. Phys. 3, 107 (1935).

[24] A. Fersht, Structure and Mechanism in Protein Science: A Guide to Enzyme Catalysis and Protein Folding (W. H. Freeman and Co., New York, 1998).

[25] Y. Skhiri, P. Gruner et al., Soft Matter 8, 10618 (2012).

[26] I. Langmuir, J. Am. Chem. Soc. 39, 1848 (1917).

[27] See Supplemental Material at http://link.aps.org/ supplemental/10.1103/PhysRevLett.112.028301 for details of materials and methods, derivation of the reaction-adsorption model and supplemental data.

[28] F. Jin, R. Balasubramaniam, and K. Stebe, J. Adhes. 80, 773 (2004).

[29] R. Kopelman, Science 241, 1620 (1988).

[30] As no fluorescence is observed at the interface, this likely corresponds to the experimental conditions.

[31] S. Narayan, J. Muldoon, M. G. Finn, V. V. Fokin, H. C. Kolb, and K. B. Sharpless, Angew. Chem., Int. Ed. Engl. 44, 3275 (2005).

[32] E. Cordes, Pure Appl. Chem. 50, 617 (1978).

[33] T. Dwars, E. Paetzold, and G. Oehme, Angew. Chem., Int. Ed. Engl. 44, 7174 (2005).

[34] C. E. Kolb and D. R. Worsnop, Annu. Rev. Phys. Chem. 63, 471 (2012).

[35] P. Luisi, The Emergence of Life from Chemical Origins to Synthetic Biology (Cambridge University Press, New York, 2006).

[36] S. Mann, Angew. Chem., Int. Ed. Engl. 52, 155 (2013). 